\title{
A Price-Aware Congestion Control Protocol for Cloud Services
}

xiaocui sun ( $\nabla$ xiaocuisun1002@hotmail.com )

Guangdong Pharmaceutical University https://orcid.org/0000-0002-4342-5733

\section{Zhijun Wang}

The University of Texas at Arlington

\section{Yunxiang Wu}

Purple Mountain Laboratories

\section{Hao Che}

The University of Texas at Arlington

\section{Hong Jiang}

The University of Texas at Arlington

\section{Research Article}

Keywords: Pricing model, cloud computing, congestion control, network utility maximization

Posted Date: April 19th, 2021

DOl: https://doi.org/10.21203/rs.3.rs-364078/v1

License: (c) (1) This work is licensed under a Creative Commons Attribution 4.0 International License. Read Full License

Version of Record: A version of this preprint was published at Journal of Cloud Computing on November 20th, 2021. See the published version at https://doi.org/10.1186/s13677-021-00271-5. 


\section{Abstract}

In current infrastructure-as-a service (laaS) cloud services, customers are charged for the usage of computing/storage resources only, but not the network resource. The difficulty lies in the fact that it is nontrivial to allocate network resource to individual customers effectively, especially for short-lived flows, in terms of both performance and cost, due to highly dynamic environments by flows generated by all customers. To tackle this challenge, in this paper, we propose an end-to-end Price-Aware Congestion Control Protocol (PACCP) for cloud services. PACCP is a network utility maximization (NUM) based optimal congestion control protocol. It supports three different classes of services (CoSes), i.e., best effort service (BE), differentiated service (DS), and minimum rate guaranteed (MRG) service. In PACCP, the desired CoS or rate allocation for a given flow is enabled by properly setting a pair of control parameters, i.e., a minimum guaranteed rate and a utility weight, which in turn, determines the price paid by the user of the flow. Two pricing models, i.e., a coarse-grained VM-Based Pricing model (VBP) and a fine-grained Flow-Based Pricing model (FBP), are proposed. The optimality of PACCP is verified by both large scale simulation and small testbed implementation. The price-performance consistency of PACCP are evaluated using real datacenter workloads. The results demonstrate that PACCP provides minimum rate guarantee, high bandwidth utilization and fair rate allocation, commensurate with the pricing models.

\section{Full-text}

Due to technical limitations, full-text HTML conversion of this manuscript could not be completed. However, the manuscript can be downloaded and accessed as a PDF.

\section{Figures}

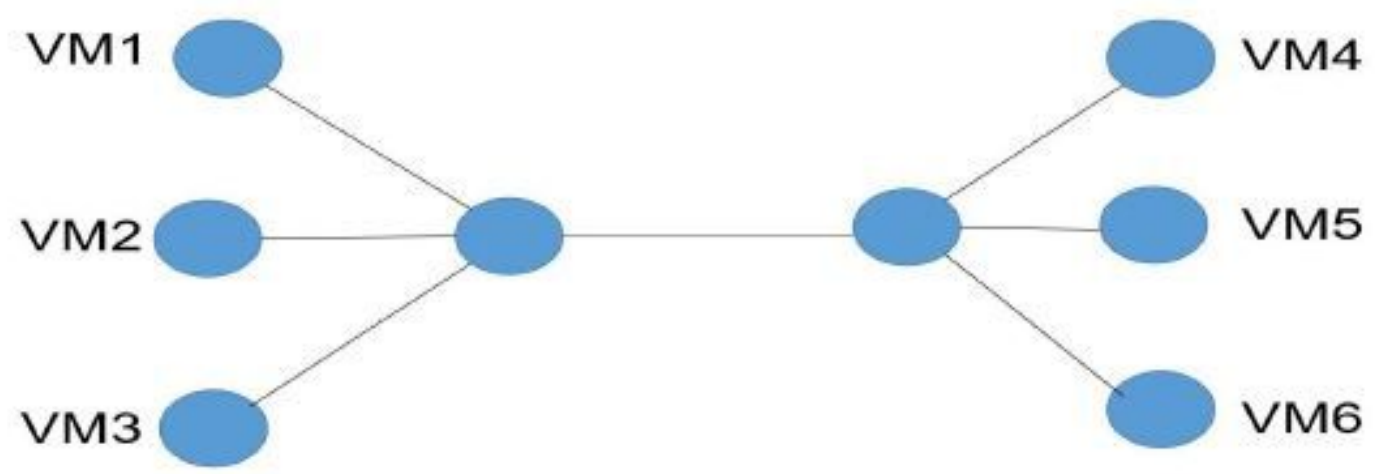

\section{Figure 1}

An example network. 


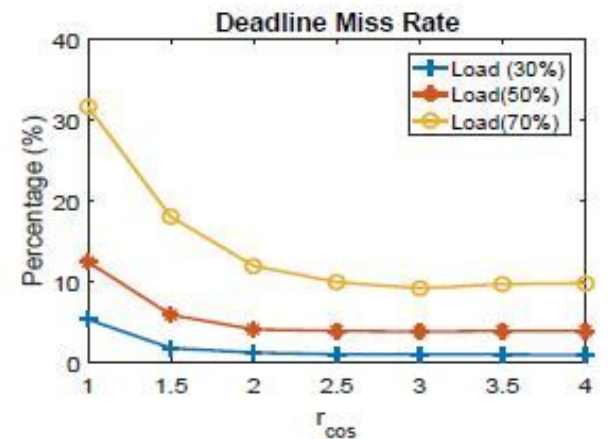

(a)

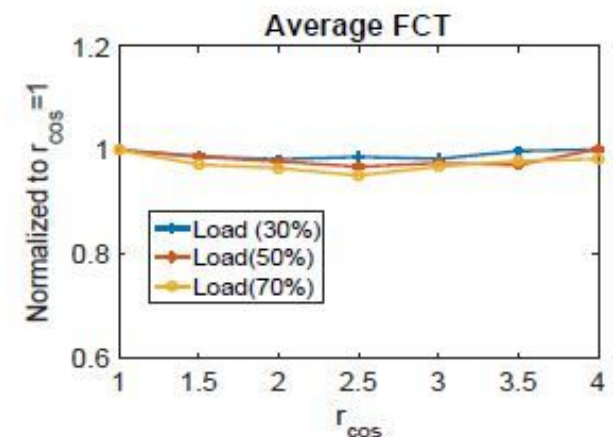

(b)

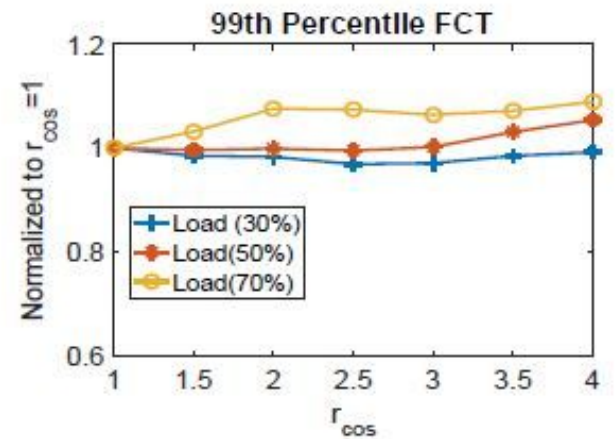

(c)

Figure 2

please see the manuscript file for the full caption

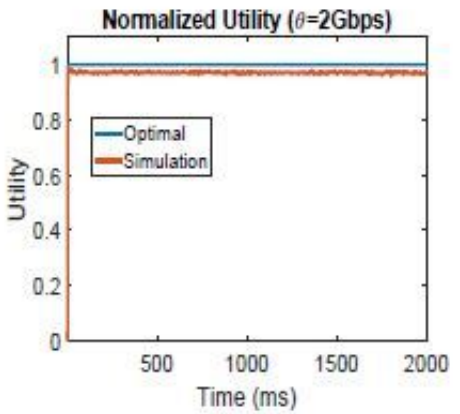

(a)

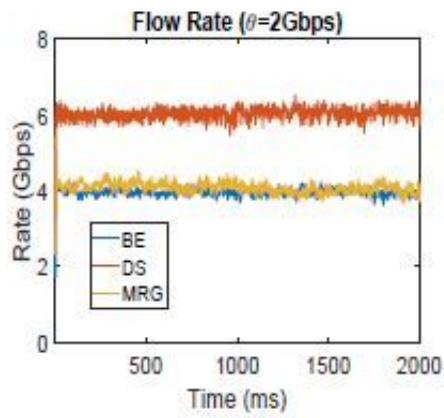

(b)

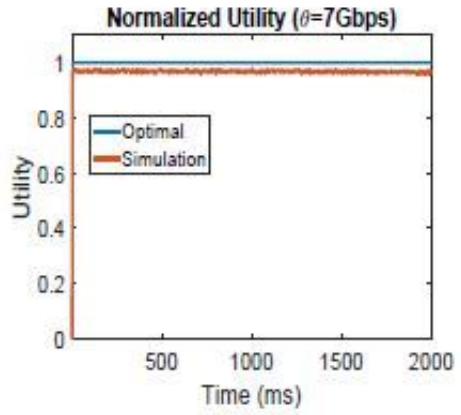

(c)

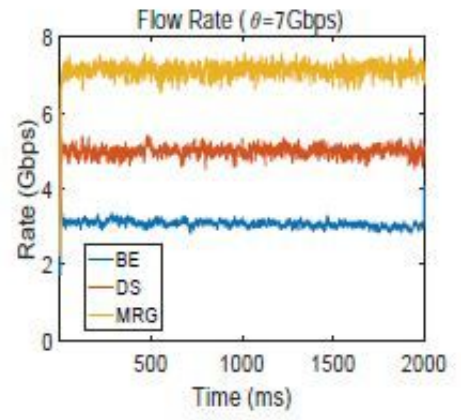

(d)

\section{Figure 3}

please see the manuscript file for the full caption

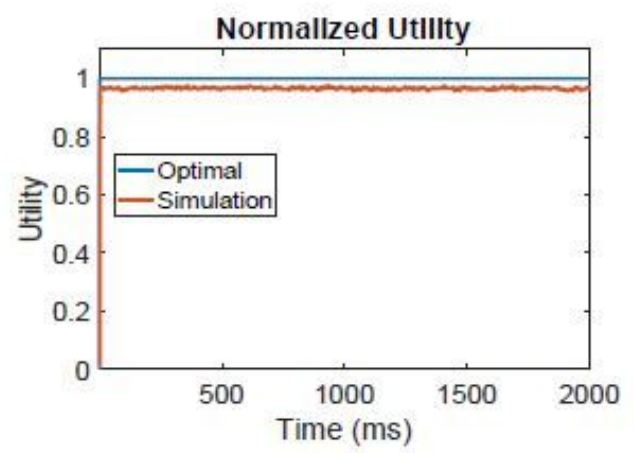

(a)

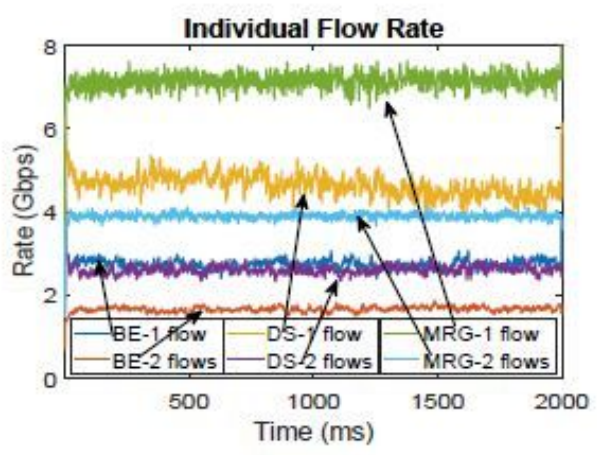

(b)

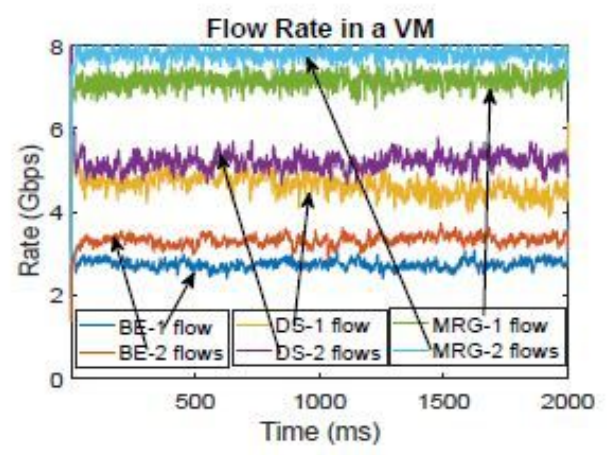

(c)

\section{Figure 4}

please see the manuscript file for the full caption 


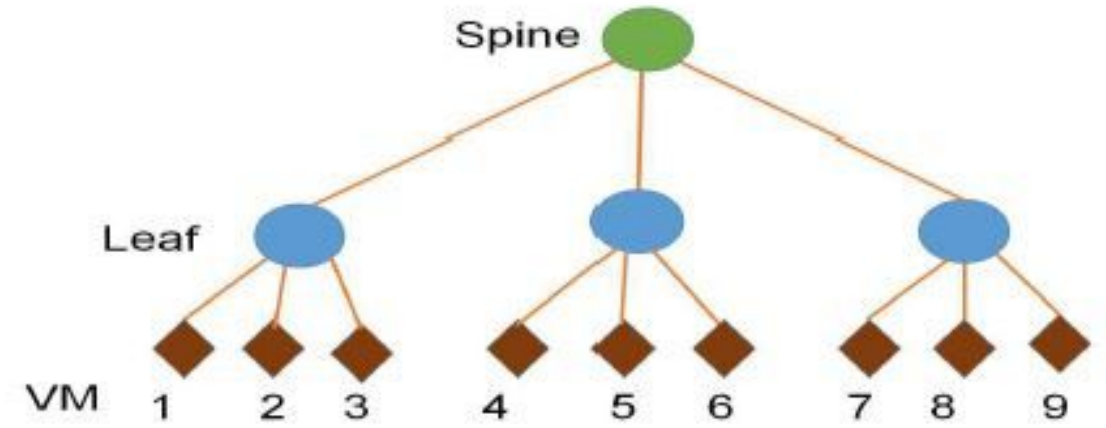

Figure 5

A 3x1 spine-leaf testbed.

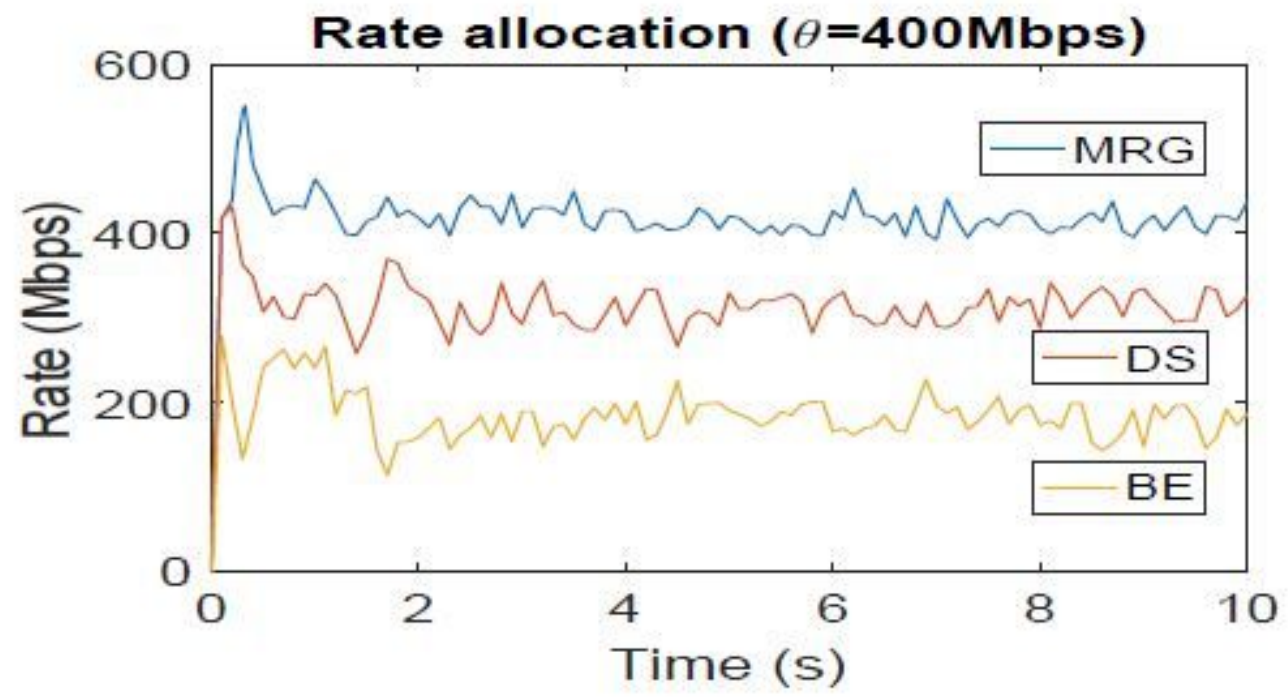

Figure 6

Rate allocation in the testbed.

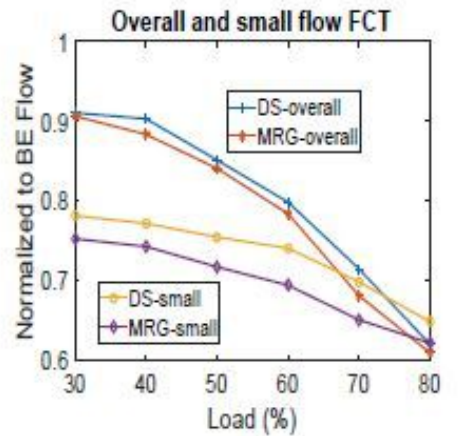

(a)

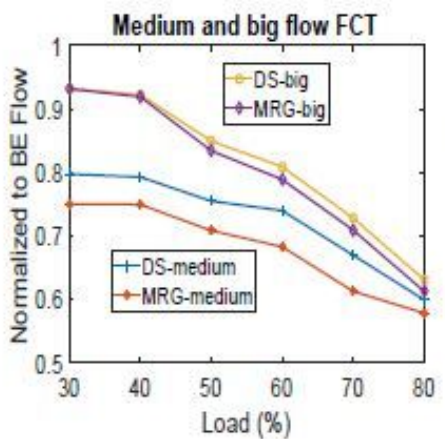

(b)

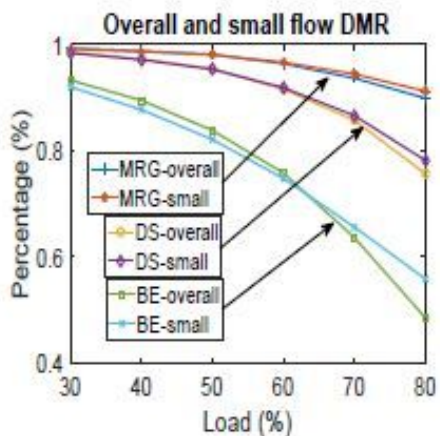

(c)

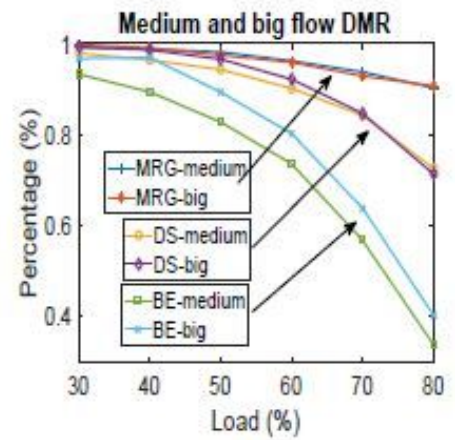

(d)

\section{Figure 7}

please see the manuscript file for the full caption 


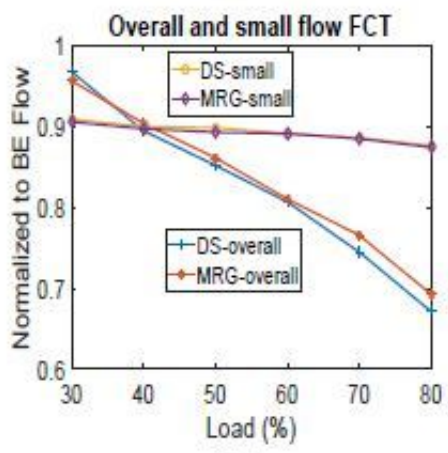

(a)

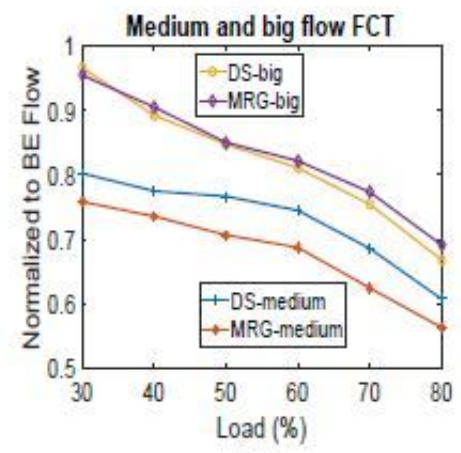

(b)

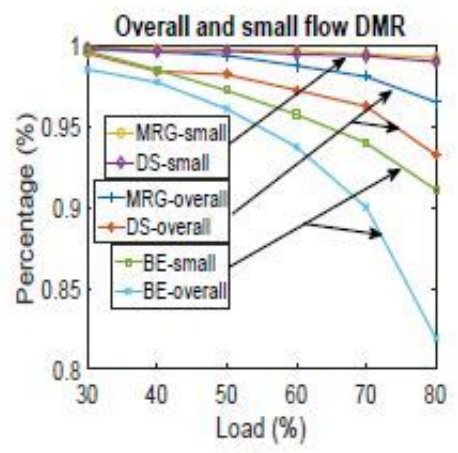

(c)

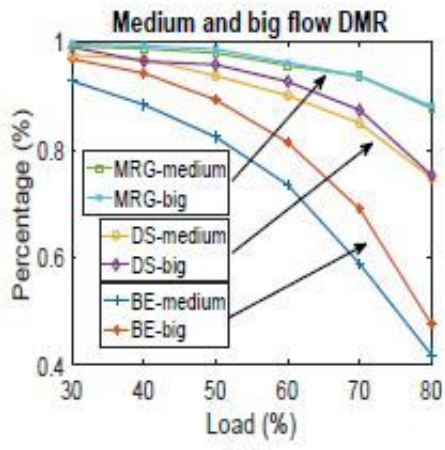

(d)

\section{Figure 8}

please see the manuscript file for the full caption

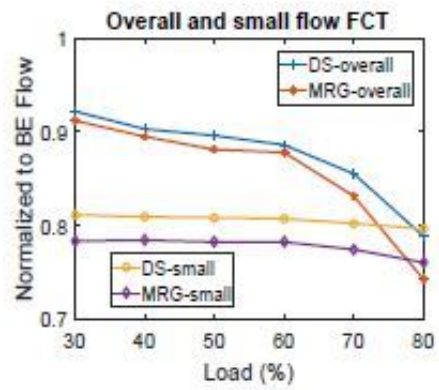

(a)

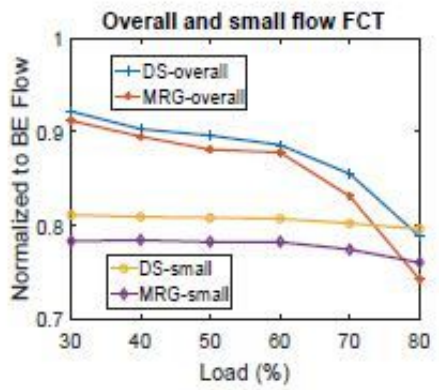

(b)

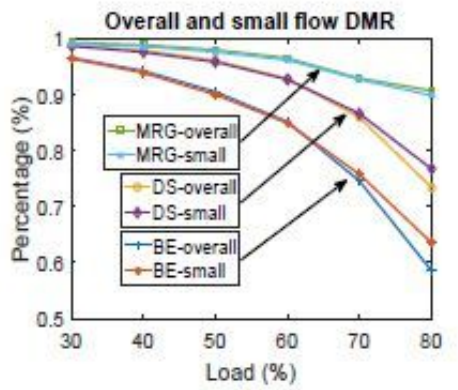

(c)

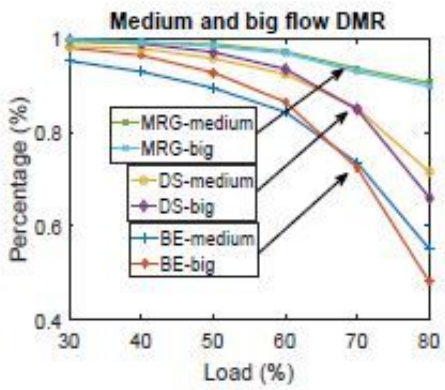

(d)

\section{Figure 9}

please see the manuscript file for the full caption

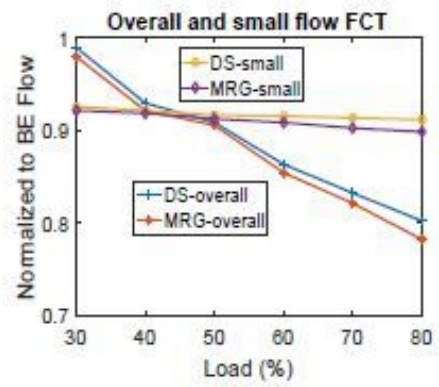

(a)

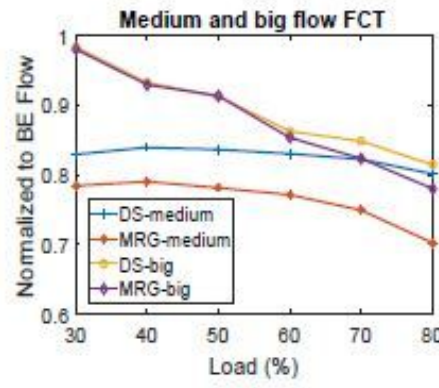

(b)

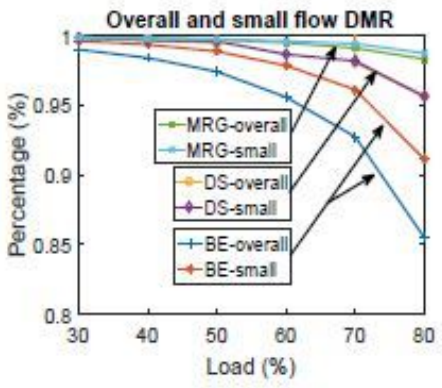

(c)

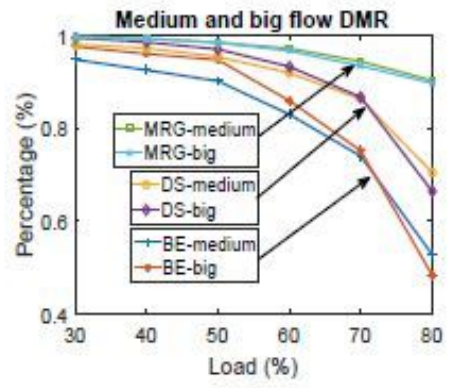

(d)

\section{Figure 10}

please see the manuscript file for the full caption 